\title{
Creatività, storia, politica: alcuni spunti sul tema
}

\section{Paolo Prodi}

Storicamente, 1 (2005).

ISSN: 1825-411X. Art. no. 62. DOI: 10.12977/stor524

[1]

Ogni Stato ha la sua politica, sia interna, sia estera. La discussione - anche nella stampa, nel consiglio di governo, in parlamento - è tanto più proficua quanto più è storica, quanto più dannosa, quanto più si basa sui dottrinarismi, su idola theatri, fori, specus, tribus.

L'importanza pratica degli studi storici sta in ciò che essi - ed essi soltanto danno allo Stato, al popolo, all'esercito, ecc. l'immagine di sé stessi.

Lo studio della storia è il fondamento della preparazione e della formazione politica. L'uomo di Stato è lo storico pratico ... [2]

Così nella seconda metà dell'Ottocento Johann Gustav Droysen poteva descrivere ai suoi studenti di Berlino - e quindi di tutto il mondo dall'alto della sua cattedra - il compito dello storico: è lo storico che costruisce l'immagine dello Stato e della società e il politico è soltanto uno storico pratico, in un rapporto analogo a quello del medico di base che cerca di applicare la terapia teorizzata dal grande anatomo-patologo. Questo è stato certamente il punto più alto raggiunto dalla nostra professione, punto al quale, ormai decaduti sia come professori universitari sia in particolare come storici, non possiamo guardare senza un pizzico di nostalgia. Le nostre discipline sono state praticamente espulse dalle facoltà dove regnavano sino a pochi anni or sono e dove si formano gli uomini destinati ad operare nella società (giurisprudenza, economia, scienze politiche) e si sono rifugiate nelle 
fortezze chiuse delle facoltà di lettere: anche qui le storie sono marginalizzate perché la dimensione storica sta scomparendo anche dall'insegnamento letterario e filosofico e tende a rinchiudersi nell'ultimo bastione (o ghetto) costituito dai corsi di laurea in storia.

In realtà gli storici sin dalle loro origini, sin dalle prime espressioni della loro arte nelle genealogie dei monarchi medio-orientali o nei racconti delle guerre delle città greche, sono sempre stati importanti come specchio della politica in funzione della legittimazione del potere. Certamente nel XIX secolo con la nascita della scienza storica nel contesto del "laboratorio borghese" [3] si è assistito all'espulsione di ogni aspetto creativo, al rinnegamento delle sue radici artistico- letterarie in favore di una disciplina che, pur non potendo assumere la metodologia delle scienze sperimentali tendeva sempre più a conformarsi ad esse per partecipare alla loro ascesa sociale: euristica, critica delle fonti, interpretazione, sistematica ecc. (per usare il linguaggio dello stesso Droysen). Parallelamente al perfezionamento scientifico dei metodi ricerca, che ha trovato la sua massima espressione nella definizione del fine ultimo della storia nella ricostruzione degli avvenimenti "come essi sono realmente accaduti", secondo la famosa definizione di Leopold von Ranke, due tendenze hanno favorito l'egemonia della cultura storica nella cultura europea. 
In primo luogo, la richiesta rivolta alla storia di fornire, nella crisi delle visioni tradizionali metafisiche e metastoriche, una spiegazione di tutto il reale, del senso del cammino dell'umanità: non mi soffermo su questo problema a cui diamo globalmente il nome di storicismo (nelle sue variopinte manifestazioni di tipo idealista o marxista) perché non è su questo punto che si deve concentrare la nostra attenzione oggi: ma va comunque detto che lo storicismo ha caratterizzato il nostro modo di pensare sino alla nostra generazione e che il suo tramonto (pensiamo alla scomparsa di Benedetto Croce - della storia come storia della libertà - dalla formazione intellettuale dei nostri giovani) lascia un vuoto di cui dobbiamo essere consapevoli e che dobbiamo ancora ricuperare.

In secondo luogo - e questo interessa più da vicino il nostro discorso - la pressione sull'uso 'politico' della storia è diventata dominante in parallelo con la crescita degli Stati nazionali moderni negli ultimi due secoli. L'insegnamento della storia è divenuto la forma fondamentale dell'educazione civica ed ha condizionato per decenni la ricerca. Senza i libri di storia difficilmente i giovani europei avrebbero ucciso e si sarebbero fatti uccidere nelle trincee contrapposte della prima guerra mondiale: elemento comune il " pro patria mori", la religione della patria. Con l'avvento dei regimi totalitari e l'oppressione ideologica, con lo sviluppo dell'arte della propaganda e della censura, il sistema è ancora più tragicamente degenerato in gran parte dell'Occidente: l'uso politico della storia è stato esplicito e diretto soprattutto a consolidare, in funzione della potenza nazionale, le concezioni di supremazia di civiltà, di razza, di classe, di missione storica.

L'accentuazione della funzione della storia come strumento di formazione e di propaganda fu forse accresciuta a partire dalla fine dell'Ottocento anche dallo sviluppo delle nuove scienze sociali come la sociologia, l'antropologia, la politologia ecc.: la perdita del monopolio di scienza della società per eccellenza provocò una vuoto che rendeva gli storici molto più soggetti allinfluenza del potere politico, espresso dai partiti sviluppati nei regimi 
democratici con il suffragio universale e dai movimenti di massa.

Intorno alla seconda guerra mondiale, nella prospettiva della shoah e della bomba atomica questa visione giustificazionista o consolatoria della storia esplode in mille frantumi. Per comprendere questa trasformazione nulla è più illuminante della famosa tesi di Walter Benjamin :

"C'è un quadro di Klee che si chiama Angelus novus. Vi è rappresentato un angelo che sembra in procinto di allontanarsi da qualcosa su cui ha fisso lo sguardo. I suoi occhi sono spalancati, la bocca è aperta, e le ali sono dispiegate. L'angelo della storia deve avere questo aspetto. Ha il viso rivolto al passato. Là dove davanti a noi appare una catena di avvenimenti, egli vede un'unica catastrofe, che ammassa incessantemente macerie su macerie e le scaraventa ai suoi piedi. Egli vorrebbe ben trattenersi, destare i morti e riconnettere i frantumi. Ma dal paradiso soffia una bufera, che si è impigliata nelle sue ali, ed è così forte che l'angelo non può più chiuderle. Questa bufera lo spinge inarrestabilmente nel futuro, a cui egli volge le spalle, mentre cresce verso il cielo il cumulo delle macerie davanti a lui. Ciò che noi chiamiamo il progresso, è questa bufera.» [4]

Nella seconda metà del Novecento sotto la spinta di queste tragedie, la crisi dello Stato-Nazione e il tramonto delle ideologie hanno portato all'eclissi non soltanto dello storicismo ma anche della storia come disciplina e aperto la strada ad una nuova creatività come possibilità di elaborare la memoria del passato senza una diretta strumentalizzazione politica ed ideologica e senza la pretesa di fornire la direzione del cammino della realtà. In questo quadro si è operata negli ultimi decenni una commistione fertile con le altre discipline che studiano l'uomo e il suo ambiente e con l'esplorazione di mondi sino allora trascurati: al centro della ricerca storiografica non sono stati più messi i grandi avvenimenti della politica, ma le vicende dell'uomo comune, le mentalità, la vita materiale, la metà sino ad allora quasi ignorata dell'umanità (la componente femminile), l'ambiente, il clima.

Su questa strada sono maturati gli sviluppi storiografici innegabili che tutti 
abbiamo sotto gli occhi e che molto spesso sono reclamizzati con patine lucenti ( la "nouvelle histoire" come la "nouvelle cuisine") che contengono in ogni caso le innovazioni più creatrici: l'egemonia della scuola francese delle Annales negli scorsi decenni è derivata certamente dall'aver saputo cogliere con grande lucidità la crisi della storiografia politica tradizionale e dall'aver proposto l' espansione dei panorami storici in una visione del divenire delle civiltà nel loro insieme .

Ma l'abbandono del primato della storia politica ha portato anche ad alcune deviazioni che hanno caratterizzato gli ultimi decenni dello scorso secolo, del Novecento.

In primo luogo l' abolizione del confine tra storia e fiction : la storia non più come ricerca della verità, di ciò che è realmente accaduto (non certo come obiettivo raggiungibile, ma nondimeno come tensione fondamentale nella ricerca), bensì come ricreazione di un passato a nostra immagine 0 trasposizione nel passato di una visione attuale del reale. Perdute le coordinate cronologiche e la necessità della dimostrazione, della prova, si ha spesso o una storia senza tempo o una narrazione fuori dal tempo venduta come storia (dato che il pubblico ha in ogni caso fame di storia). Questa può benissimo rappresentare una creatività letteraria, anche a livelli altissimi, ma non è storia. Senza riprendere la grande discussione sul contenuto narrativo e letterario della storia penso si possa dire che spesso è venuta a meno quella distinzione tra il reale e il verosimile che ne ha sempre garantito il metodo di ricerca: è logico che per comprendere una società possa essere molto più importante un'opera poetica o teatrale (pensiamo ai drammi di Shakespeare) di un volume pieno di diagrammi e tabelle, ma questa è cosa ben diversa dalla ricreazione di un medievalismo o di un rinascimentismo di maniera, anzi è l'opposto.

In secondo luogo, al servizio diretto al potere politico ed economico dello Stato nazione per la costruzione del cittadino-suddito prevalente nella prima metà del Novecento si è spesso sostituita una funzione servile o mercenaria 
come adesione alle richieste provenienti dai nuovi poteri emergenti dalla società. II revisionismo non come continua messa in questione delle nostre interpretazioni del passato (ciò che è sempre una componente fondamentale del lavoro dello storico), ma come manipolazione strumentale a sostegno dell'una o dell'altra posizione dominante nelle istituzioni, nei partiti e nel mondo del potere economico. Soprattutto in senso passivo: l'uso del linguaggio politically correct rappresenta a mio avviso il pericolo più grave in questa direzione anche per la nostra vita democratica, con l'espulsione dal racconto storico di tutte le situazioni e degli stessi vocaboli che sono ritenuti indigesti o pericolosi per i nostri attuali modelli di comportamento, offensivi per una "opinione pubblica" vista come detentrice non solo del presente, ma anche del monopolio della memoria.

Non pretendo certo di definire qui qual è oggi il mestiere dello storico ma credo si possa dire molto semplicemente che il suo scopo è quello di fornire ai propri con-uomini (oggi si usa molto parlare di "gente" ma questa parola ha assunto significati equivoci) un particolare occhiale che serve per vedere il passato che è incorporato nel mondo che ci circonda (cose e idee) e in noi stessi, passato che è invisibile ad occhio nudo. In questa età di transizione verso la globalizzazione (per esprimersi in termini rozzi ma brevi) la fame di storia esprime nelle nostre società un bisogno analogo a quello per cui si ricorre sempre più spesso alle pratiche psicanalitiche per la ricostruzione della nostra personalità individuale: un recupero delle nostre identità collettive perdute, della cui coscienza abbiamo bisogno per sopravvivere e per poter confrontarci con le altre identità [5].

In questo passaggio del millennio, oltre la modernità, con la caduta del mito del progresso come cammino sicuro dell'umanità verso una salvezza secolarizzata, in un sistema di conoscenza e di trasmissione del sapere che abolisce completamente od emargina, sotto la pressione delle nuove tecnologie informatiche la dimensione temporale, la creatività della storia sembra consistere soprattutto nel recupero di questa dimensione per far capire che le cose sono state diverse da come si presentano nella 
dimensione appiattita del presente e della cronaca e che saranno diverse anche in futuro. II tempo diventa così, insieme allo spazio, una delle dimensioni indispensabili per capire se stessi e il diverso, sfuggendo alla condanna di un presente immobile. In questo senso mi sembra che la storia stia ritrovando il suo ruolo creativo specifico all'interno delle scienze sociali: mentre queste sono per lo più preoccupate della elaborazione di modelli di comportamento, la storia punta tutte le sue carte sul mutamento e sulla molteplicità infinita delle differenze, sull'evento, su ciò che è avvenuto, come realtà particolare e irrepetibile. Creatività nel senso di avvicinarci il più possibile ad ogni traccia che l'umanità ha lasciato, ad ogni "odore di carne umana" (come diceva Marc Bloch), cercando di escludere soltanto l'uomo artificiale inventato, come trappola impagliata, per la cattura degli animaliuomini.

Per quanto riguarda il tema specifico che mi è stato dato - la politica - la creatività della storia sembra consistere soprattutto nel capire e far capire che le diagnosi e i processi decisionali basati solo sull'osservazione dell'oggi e su modelli a-temporali possono condurre a catastrofi di grandi dimensioni. Non una storia dei "se fosse... (il naso di Cleopatra...) ma una storia dei problemi e delle scelte che l'umanità ha affrontato nelle varie epoche. Oggi il problema più angoscioso è forse quello dell' uomo politico il quale non ha la possibilità e la capacità di elaborare e proporre opzioni, di essere quindi un creatore di scelte e di decisioni: non può essere un leader (nel senso etimologico come "guida") della società ma deve seguirne gli umori che si esprimono nei sondaggi; essere scelto e non scegliere è il suo dramma in tutti i paesi democratici e liberali al di là di tutte le affermazioni retoriche sui programmi.

La creatività della storia non consiste quindi nella ricostruzione del passato ma nel liberarci dall'oppressione del presente, nel conoscere le molteplici e intricate strade che abbiamo percorso: questo ci aiuta anche a capire dove stiamo andando e almeno ci dimostra che domani saremo ancora diversi. Al di là di ogni discorso teorico la cosa migliore, arrivati a questo punto, è 
presentare alcuni exempla che ci aiutino a comprendere il mutamento che si nasconde dentro alcune tra le parole più usate ed abusate in politica: si tratta naturalmente soltanto di spunti di discussione che meriterebbero una ben più approfondita trattazione, spunti ai quali ne potremmo aggiungere innumerevoli altri.

La guerra. Siamo immersi da milioni di parole ma rischiamo di non capire nulla. Per uno storico di lungo periodo parlare di "guerra" al terrorismo non ha alcun senso se questa parola è assunta nel significato acquisito durante gli ultimi secoli. Penso che una data epocale, almeno per quanto riguarda I'Italia (ma con piccole oscillazioni di un decennio per tutto l'Occidente) rimarrà il 2005 come anno in cui è finita la leva militare obbligatoria. Dalla fine dell'antico regime ma soprattutto con il formarsi delle grandi armate rivoluzionarie e napoleoniche il servizio militare obbligatorio è stato il cemento sul quale si è costruita l'identità statale-nazionale sul principio del "pro patria mori". Ciò a conclusione di alcuni secoli di un percorso in cui lo Stato moderno a poco a poco ha eliminato la violenza "diffusa" nelle società pre-moderne : pensiamo alla faida come istituto giuridico medievale, alle vendette private, alle lotte di fazione nelle nostre città ecc. A questa situazione si è sostituito lo Stato come detentore del monopolio della violenza legittima: solo lo Stato può uccidere e può permettere di uccidere rendendo anzi l'uccisione e la morte in guerra un atto sacrale. La pace ha potuto esistere soltanto come intervallo tra le guerre ed è stata negli ultimi secoli direttamente dipendente da esse: un prezzo terribile pagato quasi da ogni generazione con rituali sempre più elaborati e che si concludeva, dopo i trattati di pace, con i monumenti ai caduti che sono parte integrante del paesaggio di ogni nostro villaggio.

Tutto questo ora è finito: non vi sono più dichiarazioni di guerra né trattati di pace; anche gli Stati possono essere definiti "canaglie" e quindi non più soggetti di diritto sovrano. La bomba fabbricata in casa da un kamikaze e l'areo o missile ad altissima precisione che bombarda la casa dove questa bomba potrebbe essere stata costruita rappresentano visivamente il fatto 
che non si tratta più di guerra o di guerriglia nel senso in cui l'abbiamo inteso durante tutta l'età moderna ma di un conflitto di tipo nuovo di fronte al quale le armi e gli "ordini" giuridici tradizionali sono impotenti. Non possono non esservi ripercussioni sia nell'analisi del fenomeno "terrorismo" sia nella diagnosi sulla solidità della nostra "pace" quotidiana come adesione alle leggi dello Stato, dal pagamento delle imposte a tutto il resto. Si tratta di un ritorno alla violenza diffusa in cui analogie e differenze storiche si intrecciano: come lo sviluppo del monopolio della violenza da parte dello Stato nell'età moderna era legato al progresso tecnologico e alle nuove armi (come i cannoni ecc.) così ora le innovazioni tecnologiche rendono vulnerabili le società attuali e impossibili le guerre in senso tradizionale. Ciò con buona pace dei pacifisti totali e dei neoconservatori, uniti nel mito di un mondo democratico che per sua virtù intrinseca annulli la violenza: con questo non si vuole certo negare il valore pedagogico delle manifestazioni contro la guerra, ma chiedere che, sino a quando non si sia delineato un nuovo ordine mondiale per il superamento dei conflitti, siano attivati gli strumenti necessari per impedire che la violenza, rotti gli argini precedenti, si diffonda nella società.

Più in profondità, infatti, il problema è quello di capire quali sono state le strade seguite e quali sono possibili ora per controllare la violenza come parte insopprimibile della natura umana. Da qui innumerevoli corollari: quali possono essere i surrogati per la funzione civile che gli eserciti hanno indubbiamente svolto. Pensiamo, per fare un piccolo esempio, al peso che in Italia ha avuto la "naia" per la formazione e la pratica della cittadinanza; pensiamo alle funzioni civiche esercitate dalle associazioni di ex combattenti per la solidarietà nazionale (es. l'Associazione Nazionale Alpini). Pensiamo, in senso opposto, al peso che ha e che avrà ancor più nel mondo come in Italia la formazione di eserciti professionali. Le compagnie di ventura e i mercenari hanno costituito lo strumento di passaggio dagli eserciti feudali al moderno: ora le guerre sono affidate a specialisti "soldati" cioè assoldati nel senso etimologico del termine o addirittura appaltate a ditte. Nulla è più 
eccitante che il ripercorrere questo cammino per comprendere il presente. Anche il bellissimo film di Ermanno Olmi "Il mestiere delle armi" si è mosso con intuizione poetica in questa direzione.

Sacro e potere, Chiesa e Stato. Nella lunga storia dell'Occidente questi due binomi sono stati sempre ritenuti equivalenti. Ora essi sono dissociati: il potere e il sacro vagano senza recinti. Naturalmente anche qui non si tratta di un processo di breve periodo: i totalitarismi del XX secolo ci appaiono sempre di più come religioni secolarizzate, come primo esperimento di questa rottura, di un sacro uscito dall'otre delle Chiese in cui con tante fatiche e tante tragedie esso era stato rinchiuso nei secoli precedenti. Nessuna visione idilliaca anche a questo proposito: le radici liberali dell'Occidente affondano in queste tensioni, in queste controversie interminabili in cui il sacro ha sempre cercato di impadronirsi del potere politico e viceversa ma proprio attraverso queste dialettiche si erano in qualche modo creati dei recipienti di contenimento, contenitori rotti dalle ideologie totalitarie tese ad impadronirsi di tutto l'uomo.

Ma noi, attestandoci su posizioni laiciste o clericali che spesso non si preoccupano d'altro che dei relitti del passato, continuiamo a parlare di fondamentalismi, di Islam estremista o moderato, di civiltà cristiana come se nulla fosse successo. In realtà dal punto di vista della storia di lungo periodo I'Islam ci appare come un'eresia giudaico-cristiana che non ha seguito l'Occidente nel faticoso processo di distinzione del sacro dal potere, che non conosce la Chiesa come istituzione, come diversa dallo Stato. Se questo è vero, il problema è principalmente nostro: l'azione di supporto e sostegno alla modernizzazione del mondo islamico deve essere accompagnata dalla soluzione del nostro problema sul come salvare nelle nuove situazioni storiche il dualismo tra sacro e potere che costituisce il DNA della nostra civiltà, la vera radice dell'Europa e dell'Occidente, dualismo che sta scomparendo dal nostro panorama culturale, politico e giuridico. Di fronte ai problemi della difesa dei viventi e dell'ambiente, delle manipolazioni genetiche, della scarsità delle risorse l'impotenza del diritto statale positivo, 
della "norma ad una dimensione" è evidente. Cosa proponiamo? Basta l'appello alla coscienza personale di fronte all'incarnazione nei nuovi grandi poteri economici? Basta, in senso opposto, definire come reato giuridico ogni deviazione da un'etica dominante ma non condivisa? In rapporto all'angolo di osservazione storica assunto cambia la visione del bagaglio che come uomini occidentali portiamo con noi affrontando i problemi della globalizzazione, nel cui contesto, al di là dell'Islam si affacciano altre civiltàreligioni, come il confucianesimo e l'induismo, nelle quali non si pone il problema della coscienza e della salvezza individuale, della scelta tra il bene e il male che caratterizza in un modo o nell'altro gli eredi delle religioni monoteiste nate intorno al Mediterraneo.

Democrazia e rappresentanza. Siamo frastornati dal rumore delle polemiche sulla crisi dei partiti, sulla fragilità dei governi, sul calo della partecipazione dei cittadini, sui rimedi possibili; progetti di riforma dei meccanismi elettorali vengono proposti e riproposti, dal proporzionale al maggioritario e in senso inverso, senza soste, con grande impegno dei politologi, in un vai e vieni frenetico. La storia di lungo periodo ci insegna che il nostro sistema basato sui partiti, sul collegio elettorale, sulla legislatura parlamentare è nato nell'Inghilterra del Settecento ancor prima dell'era della ferrovia. Ora le coordinate spaziali e temporali, che stavano alla base di questo sistema e che bene o male avevano retto sino a qualche anno fa, sono crollate: le distanze sono annullate e il ritmo del tempo è completamente diverso. Una visione storico critica porta a capire che tutte le riforme progettate dai politologi sono solo palliativi e che è assolutamente necessario per salvare la democrazia inventare forme del tutto nuove di partecipazione. Non è sufficiente lamentarsi dello svuotamento dei poteri delle nostre assemblee rappresentative, di una politica condotta attraverso gli schermi televisivi. Le scelte fondamentali che l'umanità deve compiere nel prossimo futuro sono del tutto incompatibili con gli spazi e i tempi elettorali del presente: sia nella necessità di rapidità dei processi decisionali sia - ciò che è ancora più importante - perché le grandi scelte come quelle relative alle tematiche 
genetiche, alle fonti di energie, al controllo delle risorse del pianeta, allo smaltimento dei rifiuti riguardano le generazioni future e molto spesso sono in netto contrasto con gli interessi elettorali del momento, al di là delle divisioni e dei programmi politici. La democrazia, pur così vitale, dei nostri comuni medievali, morì quasi sul nascere per il contrasto tra l' esigenza della partecipazione popolare (pur così forte e culturalmente fondata) da una parte e i tempi e gli spazi della vita quotidiana e delle istituzioni cittadine dall'altra: quando la durata della Signoria era in Firenze di due mesi e il consiglio cittadino era composto da migliaia di persone tutte legittimamente indaffarate nel lavoro e nella cura dei propri interessi. Girolamo Savonarola fece costruire la sala dei Cinquecento per poter stabilire un governo popolare, per permettere che il Consiglio grande ( di circa 3600 persone ) potesse riunirsi almeno a turno. Fu un grande sforzo ma anche una grande sconfitta: vinse il principato perché più funzionale alla vita di quel tempo. $\mathrm{E}$ Savonarola fu bruciato sul rogo il 23 maggio 1498 [6].

Federalismo ed Europa . Forse è il punto su cui maggiormente sentiamo in questi giorni la mancanza di un vero discorso storico, della creatività che soltanto la storia di lungo periodo può darci. Da una parte l'invenzione di realtà immaginarie come la Padania, dall'altra la presentazione del problema come se dovessimo riprendere l'opzione del momento storico dell'unificazione italiana, degli anni Sessanta dell'Ottocento, quando l'Italia optò per lo Stato accentrato rifiutando l'ipotesi federale: come se potessimo ritornare ad un bivio e imboccare la strada allora non scelta. In realtà lo Stato sovrano non esiste più mentre le Regioni proposte assomigliano sempre più ai vecchi staterelli pre-unitari o a modelli stranieri che stanno mostrando la loro debolezza (del resto la struttura federale non riuscì a impedire in Germania l'ascesa del totalitarismo nazista). Se la crisi non investe soltanto il modello di Stato italiano ma oltre questo anche lo Stato sovrano, costruito nei secoli dell'età moderna, allora la diagnosi è completamente diversa e diverso deve essere il nostro approccio. Sia il cammino centralista che quello federale supponevano infatti il monopolio 
della sovranità, la presenza di un potere originario di dominio su di un territorio: in questo quadro il federalismo rappresentava soltanto una diversa distribuzione, all'interno di un unico ordinamento, della validità delle singole norme come cammino verso la razionalizzazione e la modernità delle istituzioni. I fenomeni attuali di mondializzazione da una parte e di regressione a comportamenti localistici, etnici e razziali dall'altra impongono un'impostazione del tutto diversa che esige per impedire disastri la definizione di diversi livelli di identità collettive culturali e politiche, dal comune all'Europa, realtà legate tra loro da nuovi patti politici più vicini a quelli che hanno portato nel medioevo alla confederazione svizzera ( Eidgenossenschaft) che non alle moderne costituzioni scritte. Come immaginiamo noi l'Europa? Come uno Stato che a poco a poco assorba la sovranità degli Stati aderenti portandola ad un livello più ampio senza intaccarla? Come un grande Stato federale a tre livelli? lo penso che i punti di snodo e di rottura tra le nuove forze centrifughe e centripete del potere tenderanno a disporsi su linee ben diverse da quelle degli ultimi due secoli. In ogni caso il superamento della storia di quest'ultimo periodo per una conoscenza dell'età che precede lo Stato moderno può costituire un buon fondamento e un buon allenamento per la nostra fantasia. I giuramenti collettivi dei nostri comuni e i patti di "signoria" hanno costruito la prima tappa del cammino europeo.

Concludendo possiamo affidare agli uditori due diverse riflessioni .

In termini poetici-apocalittici padre David Maria Turoldo diceva qualche anno fa: «Profeta non è chi annuncia il futuro ma chi denuncia il presente. Denunciare il presente vuol dire confrontare la realtà con la parola eterna, con la parola che non delude mai. Non perdersi in calcoli e logiche di potere...». Seguendo questa intuizione su di un piano più secolare (nel senso anche di non uscire dal secolo, dal tempo) lo storico è colui che confronta la realtà attuale non con la "Parola" immobile ma con ciò che è avvenuto e divenuto: non un profeta ma uno che, se riesce ad essere libero dai condizionamenti, può farci vedere spessori della realtà non percepibili ad 
occhio nudo, togliere le maschere del potere e contribuire a far crescere la coscienza dell'umanità e quindi la sua libertà.

In termini più terrestri uno degli storici maggiori del nostro tempo, Carlo Maria Cipolla, così scriveva presentando il suo saggio satirico Le leggi fondamentali della stupidità umana :

«Le faccende umane si trovano, per unanime consenso, in uno stato deplorevole. Questa peraltro non è una novità. Per quanto indietro si riesca a guardare, esse sono sempre state in uno stato deplorevole. II pesante fardello di guai e di miserie che gli esseri umani devono sopportare, sia come individui che come membri della società organizzata, è sostanzialmente il risultato del modo estremamente improbabile - e oserei dire stupido - in cui la vita fu organizzata sin dai suoi inizi» [7].

Anche la missione di conoscere e, se possibile, attenuare la stupidità umana non è davvero cosa da poco per uno storico. Un collega ed amico tedesco, Wolfgang Reinhard, ha definito recentemente la funzione dello storico nel momento attuale come "delegittimazione": se è sufficientemente indipendente, lo storico, con la sua ricerca sempre incompiuta e relativa, può dire verità che mettono in questione le certezze dei detentori del potere, come gli antichi buffoni di corte scoprivano la nudità del sovrano [8].

\section{Note}

[1] Testo della relazione presentata al Convegno internazionale sulla innovazione e la creatività

(<http://www.nuovoeutile.it/index.php?cat=3\&lang=ita>), Firenze settembre 2004.

[2]J. G. Droysen, Istorica: lezioni sulla enciclopedia e metodologia della storia, trad.it. Milano-Napoli, Ricciardi, 1966, 372.

[3]P. Schiera, I/ laboratorio borghese. Scienza e politica nella Germania dell'Ottocento, 
Bologna, il Mulino, 1987.

[4] W. Benjamin, Sul concetto di storia, Torino, Einaudi, 1997, 35-37.

[5]P. Prodi, W. Reinhard (eds.), Identità collettive tra medioevo ed età moderna, Bologna, Clueb, 2002.

[6] P. Prodi, Gli affanni della democrazia. La predicazione del Savonarola durante l'esperienza del governo popolare, in G.C. Garfagnini (ed.), Savonarola e la politica, Firenze, ed. del Galluzzo, 1997, 27-74.

[7]C.M. Cipolla, Allegro ma non troppo, Bologna, il Mulino, 1988, 43.

[8] W. Reinhard, Geschichte als Delegitimation (discorso tenuto a Monaco di Baviera il 23 novembre 2001 in occasione del conferimento del "Preis des Historischen Kolleg" alla presenza del presidente della Repubblica Federale Tedesca Johannes Rau), in "Jahrbuch des Historischen Kollegs 2002", pp. 27-37. 